\title{
Concurrent TP53 mutations predict poor outcomes of EGFR-TKI treatments in Chinese patients with advanced NSCLC
}

This article was published in the following Dove Press journal: Cancer Management and Research

\author{
Helei Hou* \\ Kang Qin* \\ Yu Liang \\ Chuantao Zhang \\ Dong Liu \\ Haiping Jiang \\ Kewei Liu \\ Jingjuan Zhu \\ Hongying Lv \\ Tianjun Li \\ Xiaochun Zhang
}

Department of Medical Oncology, The Affiliated Hospital of Qingdao University, Qingdao, Shandong Province, People's Republic of China

*These authors contributed equally to this work
Correspondence: Xiaochun Zhang Department of Medical Oncology, The Affiliated Hospital of Qingdao University, Qingdao University, 16 jiangsu Road, Qingdao, Shandong Province 266005, People's Republic of China

Tel +8605328 29I 327I

Fax +8 $60532829|327|$

Email zhangxiaochun9670@I26.com
Purpose: The study investigated the impact of TP53 mutations on the clinical efficacy of first-generation EGFR-tyrosine kinase inhibitors (TKIs) in Chinese patients with advanced or recurrent non-small-cell lung cancer (NSCLC).

Patients and methods: Tissues from 163 NSCLC patients at the Affiliated Hospital of Qingdao University were analyzed by next-generation sequencing (NGS) to determine the mutational status of EGFR and concurrent genetic alterations. TP53 mutations were evaluated in relation to baseline patient characteristics and treatment outcomes (progression-free survival [PFS], overall survival [OS], objective response rate [ORR] and disease control rate [DCR]).

Results: Among 163 patients with advanced NSCLC, 77 were identified as EGFR-mutant (47.2\%). Six patients who did not receive TKI treatment were excluded. Among the remaining 71 patients with EGFR genetic alterations, the frequency of pathogenic TP53 mutations was $60.6 \%(43 / 71)$, while other concurrent mutations were rare events. Markedly shorter median PFS (mPFS) (6.5 versus 14.0 months, $P=0.025)$ and median OS (mOS) $(28.0$ versus 52.0 months, $P=0.023$ ) were observed in TP53-mut patients than in TP53-wt controls. The overall DCR and ORR of TP53-mutant patients were both lower than those of the TP53wt cases (DCR: $76.7 \%$ versus $89.3 \%, P=0.160$; ORR: $25 \%$ versus $28 \%, P=0.374$ ). Differences in prognosis were significant, especially in the subgroup of patients with TP53 non-missense mutations, non-disruptive mutations, mutations in exon 6 , mutations in exon 7 and mutations in the non-DBD region among all TP53 mutations.

Conclusion: TP53 mutations reduce responsiveness to TKIs and worsen the prognosis of EGFR-mutant NSCLC patients, especially for those with non-missense mutations and nondisruptive mutations, as well as mutations in exon 6 , exon 7 and non-DBD region, thus acting as an independent predictor of poor outcome in advanced NSCLC patients treated with first-generation TKI therapy. Our study also suggests that TP53 mutation might be involved in primary resistance to EGFR-TKIs in Chinese NSCLC patients.

Keywords: TP53, epidermal growth factor receptor, tyrosine kinase inhibitors, non-smallcell lung cancer, mutation, exon

\section{Introduction}

Tumor suppressor gene TP53 is the most frequently mutated gene $(>50 \%)$ in human cancers. It is located on the short arm of chromosome 17 (17p13.1) in humans and has been regarded as the "guardian of the genome" because of its role in conserving stability and preventing genome mutations. ${ }^{1,2}$ It consists of 11 exons and encodes tumor protein p53, which is a 393-aa protein with three distinct domains: the 
transactivation domain, the DNA-binding domain (DBD) and the C-terminal domain. The DBD is encoded by exons 5-8, which comprises residues 102-292 and recognizes a consensus sequence in the promoter region of several genes that are associated with DNA repair, cell cycle arrest, senescence and/or apoptosis. The sequencespecific transcriptional activity mediated by DBD accounts for the principal mechanism of the tumor-suppressing function of protein p53. ${ }^{3}$ About $70-80 \%$ of TP53 gene mutations are missense mutations confining the DBD region of gene TP53, and over $90 \%$ of the TP53 point mutations are in the highly conservative $175,245,248$, 249, 273, 282 sites. ${ }^{4,5}$ Disruption of p53's normal function possibly leads to malignant cell transformation and cancer formation. ${ }^{1,3,6}$

Non-small-cell lung cancer (NSCLC) is the most common type of lung cancer (80-85\%). NSCLC patients with activating EGFR mutations, mainly exon 19 deletions and exon 21 L8585R point mutation, usually show great responsiveness to first-generation EGFR tyrosine kinase inhibitors (TKIs) and are preferred over platinum-based first-line chemotherapy. ${ }^{7-9}$ However, almost all patients will undergo relapse and disease progression within 12-24 months after treatment initiation. ${ }^{10,11}$ Approximately $50 \%$ of secondary resistance to TKIs results from EGFR exon $20 \mathrm{~T} 790 \mathrm{M}$ mutation. ${ }^{12}$ In addition, $20-30 \%$ of NSCLC patients show primary resistance to EGFR-TKIs and demonstrate early disease progression (PD) during treatment, many at the first disease assessment time-point. The underlying mechanisms of this primary resistance are not fully understood. ${ }^{13}$ It was hypothesized that MET amplification, BIM polymorphisms, PIK3CA mutations, and alterations of the PIK3CA/ $\mathrm{AKT} / \mathrm{mTOR}$ pathway are involved in primary resistance and early disease progression in NSCLC patients undergoing TKI treatment. ${ }^{14-16}$

TP53 gene mutations can be found in 35-60\% of NSCLC patients, more frequently in squamous cell carcinomas and patients with a smoking history (especially the $\mathrm{G}>\mathrm{T}$ transversions). ${ }^{1,17,18}$ Multiple studies have suggested that TP53 mutation is a potential negative prognostic factor for the outcome of NSCLC patients with TKI therapy ${ }^{19-22}$ and may confer resistance to EGFR-TKIs. ${ }^{16,23-26}$ However, the prognostic and predictive values of EGFR/TP53 concurrent mutations on the efficacy of EGFR-TKIs in Chinese patients with advanced NSCLC remain largely unknown. In this study, we investigate the association between TP53 mutations, especially different mutation subtypes and sites, and outcome of treatment with EGFR-TKIs in Chinese patients with advanced EGFR mutation-positive NSCLC in order to determine whether TP53 mutations indicate poor prognosis and are involved in primary resistance to TKIs.

\section{Materials and methods}

\section{Patient characteristics and data collection}

We retrospectively identified 163 patients diagnosed with stage III-IV NSCLC at the Affiliated Hospital of Qingdao University between January 2014 to August 2018, whose tissue samples were routinely assessed for targeted genetic alterations by next-generation sequencing (NGS) before treatment of the first generation of TKIs. Patients had both baseline imaging and at least one repeated radiological examination. Baseline characteristics of the patients (age, gender, smoking history, family history, histology, Eastern Cooperative Oncology Group(ECOG) performance status, TKI options, line of treatment, current survival status, etc.) and outcomes after continuous and regular TKI medication were obtained using medical and radiographic records as well as patient follow-up information. All patients are Chinese. Response to TKI treatment and outcomes of the patients (progression-free survival [PFS], overall survival [OS], objective response rate [ORR] and disease control rate [DCR]) were analyzed based on EGFR and TP53 mutation status.

\section{EGFR and TP53 mutation screening by NGS}

The samples collected were either biopsies or surgical specimens, either fresh-frozen or paraffin-embedded. Targeted NGS was used for screening of EGFR and TP53 mutation status. The NGS-based assay has been described in detail in our previous publication. ${ }^{27}$

\section{Response evaluation}

Patients treated with EGFR-TKIs were re-examined regularly by means of CT (computed tomography) scanning, lab tests for tumor markers and, if clinically indicated, other examinations. The baseline imaging and at least one sequential evaluation after initiation of TKI treatment were performed for the radiographic response. Clinical response was determined according to RECIST (Response Evaluation Criteria in Solid Tumors) criteria version 1.1. ${ }^{28}$

\section{TP53 classification}

The structures of the TP53 gene have been studied extensively, and a variety of criteria have been used to 
categorize TP53 mutations according to their position, nature, and suspected effect on structure and activity of protein $\mathrm{p} 53$. For instance, TP53 genetic mutations could be classified into missense and non-missense mutations. Missense mutation refers to a mutation that results in a single amino acid change, and any genetic alterations other than missense mutations including nonsense mutations (introducing a stop codon), deletions, insertions (inframe or producing a frame shift) and substitutions at splice sites were defined as non-missense mutations. ${ }^{29}$ In addition, according to a study by Poeta et al, TP53 gene mutation can be classified into disruptive mutations and non-disruptive mutations based on the degree to which the function of $\mathrm{p} 53$ protein was changed. Disruptive mutations result in a complete, or almost complete loss of function of p53 protein, whereas non-disruptive mutations refer to conservative mutations or non-conservative mutations (excluding stop codons) outside the L2-L3 region that can retain some of the functional properties of $\mathrm{p} 53$ protein. $^{30}$

\section{Statistical analysis}

Descriptive statistics such as median and range, frequencies and percentages were used for the baseline characteristics of the patients. PFS was defined as the time from the start of first-generation TKIs (gefitinib/erlotinib/icotinib) treatment until disease progression or death (whichever occurred first): OS was defined as the time from the commencement of TKIs until death of any cause. The last follow-up date of our study was 31 August 2018 . Comparisons of the characteristics between the two groups as well as the associations between mutational status and response were assessed by Pearson's and Spearman's chisquared test or Fisher exact test, as appropriate. The Kaplan-Meier method was used for estimation of PFS and OS, and distribution curves between subgroups were compared with a two-sided log-rank test. Cox proportional hazards regression models were used to evaluate the associations between TP53 mutational status and PFS/OS, hazard ratios and 95\% confidence intervals (CI) were obtained. Logistic regression models were used to assess the associations between DCR/ORR and TP53 mutational status, and results from logistic regression are reported as $\mathrm{RR}$ and $95 \% \mathrm{CI}$. The two-tailed level of significance was set at 0.05 . All statistical analyses were performed with SPSS version 24.0 (IBM Corporation, Armonk, NY, USA) and plots generated with GraphPad Prism version 7.0 (GraphPad Software, La Jolla, CA, USA).

\section{Results}

\section{Patient characteristics}

Among a total of 163 patients with advanced NSCLC, EGFR mutations were identified in $77(47.2 \%)$ cases. Six patients who did not receive TKI treatment were excluded. A total of 157 baseline concurrent mutations were identified with NGS profiling, among which TP53 mutation showed the highest mutation frequency $(60.6 \%, 43 / 71)$. Other concurrent mutations were rare events: concurrent genetic alterations in RB1, NOTCH2 were observed in 7 patients (7/71, 9.9\%), concurrent genetic alterations in MYC, MTOR, BRCA2, SMAD4 were observed in 5 patients (5/ 71, 7.0\%); concurrent KMT2D, CDK4, CTNBB1, NF1, ARID1A mutations were observed in 4 patients (4/71, 5.6\%); the remaining 145 concurrent mutations were identified in no more than 3 patients. Only $54.9 \%$ (39/71) of all received first-generation TKIs (gefitinib, erlotinib, icotinib) as first-line therapy, the remaining 32 patients accepted TKIs as either second line therapy or postoperative adjuvant treatment. In this study, only 3 patients had squamous cell carcinomas, all 68 other patients had adenocarcinomas (ADC). EGFR and TP53 genetic alterations identified in 71 NSCLC patients by NGS-based assay are shown in Table S1 in detail. The baseline characteristics of the 71 EGFR-mutant NSCLC patients based on TP53 mutational status are summarized in Table 1. A significantly higher proportion of patients with shorter median progression-free survival (mPFS $<3 \mathrm{~m}, 32.6 \%$ versus $10.7 \%, P=0.035$ ) and shorter median overall survival (mOS $<6 \mathrm{~m}, 20.9 \%$ versus $10.7 \%, P=0.26$ ) were observed in the EGFR/TP53 concurrent mutation subgroup than in the TP53 wildtype cases. No statistically significant differences in other baseline characteristics were observed among these two groups.

\section{Univariate and multivariate analyses of clinical characteristics in relationship to outcome of EGFR-mutant NSCLC patients}

In the univariate analysis, TP53 concurrent mutations were a significant predictor of shorter mPFS (HR 1.86, 95\% CI 1.06-3.28, $P=0.029$ ) and mOS (HR 2.45, 95\% CI $1.10-5.43, P=0.024)$. In addition, uncommon EGFR mutations as well as non-first line TKI treatments were predictive factors for shorter OS (HR 3.24, 95\% CI 1.51-6.94, $P=0.001$ ) and PFS (HR 1.85, 95\% CI 1.05-3.25, $P=0.032$ ) respectively (Table 2 ).

Then, we performed multivariate analysis with the factors showing a $P$-value less than 0.05 in univariate analysis. It 
Table I Characteristics between TP53-mut and TP53-wt groups of patients with TKI treatments $(n=7$ I)

\begin{tabular}{|c|c|c|c|c|}
\hline & Total $(n=71)(\%)$ & TP53-mut $(n=43)(\%)$ & TP53-wt $(n=28)(\%)$ & $P$-value \\
\hline $\begin{array}{l}\text { Age (year) } \\
\quad \text { Median (range) }\end{array}$ & $6 I(3 \mid-78)$ & $62(31-78)$ & $61(47-77)$ & 0.657 \\
\hline $\begin{array}{c}\text { Gender } \\
\text { Female } \\
\text { Male }\end{array}$ & $\begin{array}{l}49(69.0) \\
22(30.1)\end{array}$ & $\begin{array}{l}28(65.1) \\
15(34.9)\end{array}$ & $\begin{array}{l}2 I(75.0) \\
7(25.0)\end{array}$ & 0.386 \\
\hline $\begin{array}{l}\text { Smoking status } \\
\text { Smoker } \\
\text { Never smoker }\end{array}$ & $\begin{array}{l}12(16.9) \\
59(83.1)\end{array}$ & $\begin{array}{l}9(20.9) \\
34(79.1)\end{array}$ & $\begin{array}{l}3(10.7) \\
25(89.3)\end{array}$ & 0.242 \\
\hline $\begin{array}{c}\text { ECOG PS } \\
0-1 \\
2-\end{array}$ & $\begin{array}{l}64(90.1) \\
7(9.9)\end{array}$ & $\begin{array}{l}38(88.4) \\
5(11.6)\end{array}$ & $\begin{array}{l}26(92.9) \\
2(7.1)\end{array}$ & 0.524 \\
\hline $\begin{array}{l}\text { Line of therapy } \\
\text { First line } \\
\text { Non-first line }\end{array}$ & $\begin{array}{l}39(54.9) \\
32(45.1)\end{array}$ & $\begin{array}{l}24(55.8) \\
19(44.2)\end{array}$ & $\begin{array}{l}15(53.6) \\
13(46.4)\end{array}$ & 0.856 \\
\hline $\begin{array}{l}\text { PFS (month) } \\
0-3 \\
3-\end{array}$ & $\begin{array}{l}17(23.9) \\
54(76.1)\end{array}$ & $\begin{array}{l}14(32.6) \\
29(67.4)\end{array}$ & $\begin{array}{l}3(10.7) \\
25(89.3)\end{array}$ & $0.035^{*}$ \\
\hline $\begin{array}{l}\text { OS (month) } \\
0-6 \\
6-\end{array}$ & $\begin{array}{l}12(16.9) \\
59(83.1)\end{array}$ & $\begin{array}{l}9(20.9) \\
34(79.1)\end{array}$ & $\begin{array}{l}3(10.7) \\
25(89.3)\end{array}$ & 0.26 \\
\hline
\end{tabular}

Note: $* P<0.05$

Abbreviations: TKI, tyrosine kinase inhibitor; PFS, progression-free survival; OS, overall survival; ECOG-PS, Eastern Cooperative Oncology Group performance status.

was confirmed that genetic alterations in TP53 were independently associated with shorter PFS (HR 1.87, 95\% CI 1.06-3.29, $P=0.03$ ) and OS (HR 2.73, 95\% CI 1.20-6.21, $P=0.017$ ). Uncommon EGFR mutations ( $P=0.032$ for PFS, $P=0.002$ for OS) and non-first line treatment ( $P=0.017$ for PFS, $P=0.825$ for OS) were also independent predictors of poor prognosis and reduced PFS and OS (Table 3 ).

\section{TP53 mutational status and response to TKIs}

Compared with 28 TP53-wt patients, mPFS and mOS of 43 TP53-mut patients were significantly shorter (mPFS: 6.5 versus 14 months, $P=0.025$, HR $1.86,95 \%$ CI 1.06-3.28, $P=0.029$; mOS: 28 versus 52 months, $P=0.023$, HR $2.45, \quad 95 \%$ CI $1.10-5.43, \quad P=0.024)$ (Figure 1A and B). Additionally, in patients with a PFS shorter than 3 months, $82.4 \%$ (14/17) carried TP53 mutations compared to $53.7 \%(29 / 54)$ in patients whose PFS was longer than 3 months $(P=0.002)$. In patients showing an OS shorter than 6 months, 75\% (9/12) carried EGFR mutations compared to $57.6 \%$ in patients whose OS was longer than 6 months $(P=0.05)$, indicating a high prevalence of TP53 mutations in patients presenting early resistance to TKIs (Table 1).

Since both univariate and multivariate analysis demonstrated that uncommon EGFR mutations and non-first line EGFR-TKI treatment were also predictors for worse outcomes, we further stratified patients according to type of EGFR mutations (classic EGFR Del19/L858R mutations or uncommon EGFR mutations) and treatment line (first line or non-first line), and compared the survival times of patients. Markedly shorter PFS (5.0 versus 12.0 months, $P=0.067)$ and OS (14.0 versus 52.5 months, $P=0.001)$ were observed in patients with uncommon EGFR mutations than in patients with EGFR exon 19 deletion or L858R point mutations. In the subgroup of patients with first-line EGFR-TKI therapy, the MPFS was 6.1 months longer than that of patients who received EGFR-TKIs in the non-first line setting (8.4 versus 14.5 months, $P=0.055$ ); however, no significant difference was identified in OS between these two groups of patients ( 39.0 versus 40.0 months, $P=0.567$ ) (Table 4).

We further investigated the prognostic effect of TP53 mutations on outcome of patients in each subgroup mentioned above. It was shown that concurrent TP53 mutation 
Table 2 Univariate analyses of selected factors for PFS and OS of EGFR-mut NSCLC patients (N=7I)

\begin{tabular}{|c|c|c|c|c|c|}
\hline \multirow[t]{2}{*}{ Characteristics } & \multirow[t]{2}{*}{$\mathbf{N}$} & \multicolumn{2}{|l|}{ PFS } & \multicolumn{2}{|l|}{ OS } \\
\hline & & HR $(95 \% \mathrm{CI})$ & $P$-value & HR $(95 \% \mathrm{CI})$ & $P$-value \\
\hline Age & 71 & $0.99(0.97-1.02)$ & 0.529 & $1.00(0.97-1.05)$ & 0.705 \\
\hline $\begin{array}{l}\text { Gender } \\
\text { Male } \\
\text { Female }\end{array}$ & $\begin{array}{l}22(31.0) \\
49(69.0)\end{array}$ & $\begin{array}{l}\text { I } \\
0.99(0.56-1.77)\end{array}$ & 0.979 & I.05(0.48-2.30) & 0.912 \\
\hline $\begin{array}{l}\text { Smoking status } \\
\text { Smoker } \\
\text { Non-smoker }\end{array}$ & $\begin{array}{l}12(16.9) \\
59(83.1)\end{array}$ & $\begin{array}{l}\text { I } \\
0.56(0.27-I .19)\end{array}$ & 0.128 & $\begin{array}{l}\text { I } \\
0.62(0.25-1.54)\end{array}$ & 0.298 \\
\hline $\begin{array}{c}\text { ECOG PS } \\
0-1 \\
\geq 2\end{array}$ & $\begin{array}{l}64(90.1) \\
7(9.9)\end{array}$ & $\begin{array}{l}\text { I. } 10(0.44-2.77)\end{array}$ & 0.838 & I. $.40(0.48-4.05)$ & 0.538 \\
\hline $\begin{array}{l}\text { TP53 mutation } \\
\text { Wt } \\
\text { Mut }\end{array}$ & $\begin{array}{l}28(39.4) \\
43(60.7)\end{array}$ & $\begin{array}{l}1 \\
1.86(1.06-3.28)\end{array}$ & $0.029 *$ & $\begin{array}{l}\mathrm{I} \\
2.45(1.10-5.43)\end{array}$ & $0.024 *$ \\
\hline EGFR mutation & & & 0.838 & & $0.001 *$ \\
\hline Dell 9/L858R & $5 \mathrm{I}(7 \mid .8)$ & 1 & & 1 & \\
\hline Uncommon mutations & $20(28.2)$ & $1.66(0.91-3.02)$ & & $3.24(1.5 I-6.94)$ & \\
\hline Line of therapy & & & $0.032 *$ & & 0.076 \\
\hline First line & $39(54.9)$ & I & & I & \\
\hline Non-first line & $32(45.1)$ & $1.85(1.05-3.25)$ & & I.II(0.53-2.33) & \\
\hline
\end{tabular}

Note: $* P<0.05$.

Abbreviations: NSCLC, non-small-cell lung cancer; PFS, progression-free survival; OS, overall survival; ECOG-PS, Eastern Cooperative Oncology Group performance status.

was associated with shorter mPFS in all four subgroups of patients compared to that of TP53 wild type cases (Table 4, Figure 1C and D). In terms of OS, it was shown that in the subgroup of patients with uncommon EGFR mutation, the mOS of patients with TP53 concurrent mutations was 29.5 months shorter than that of TP53 wild type cases ( 10.5 versus 40.0 months, $P=0.137$ ). In the subgroup of patients with first-line EGFR-TKI therapy, the mOS of patients with TP53 concurrent mutations was 18.8 months shorter than that of TP53 wild type cases $(10.5$ versus 40.0 months, $P=0.398$ ) (Table 4 ). But data regarding OS of patients with EGFR Del19/L858R mutation and

Table 3 Multivariate analyses of selected factors for PFS and OS of EGFR-mut NSCLC patients (N=7I)

\begin{tabular}{|c|c|c|c|c|}
\hline & \multicolumn{2}{|l|}{ PFS } & \multicolumn{2}{|l|}{ OS } \\
\hline & HR (95\% CI) & $P$-value & HR $(95 \% \mathrm{CI})$ & $P$-value \\
\hline TP53 mutation & & $0.03^{*}$ & & $0.017^{*}$ \\
\hline Wt & I & & I & \\
\hline Mut & I.87(1.06-3.29) & & $2.73(1.20-6.21)$ & \\
\hline EGFR mutation & & $0.032 *$ & & $0.002^{*}$ \\
\hline Dell 9/L858R & I & & I & \\
\hline Other mutation & $1.98(1.06-3.70)$ & & $3.58(I .6 I-7.94)$ & \\
\hline Line of therapy & & $0.017^{*}$ & & 0.825 \\
\hline First line & I & & I & \\
\hline Non-first line & $2.02(1.14-3.58)$ & & $1.09(0.5 I-2.32)$ & \\
\hline
\end{tabular}

Note: $* P<0.05$.

Abbreviations: NSCLC, non-small-cell lung cancer; PFS, progression-free survival; OS, overall survival 
A

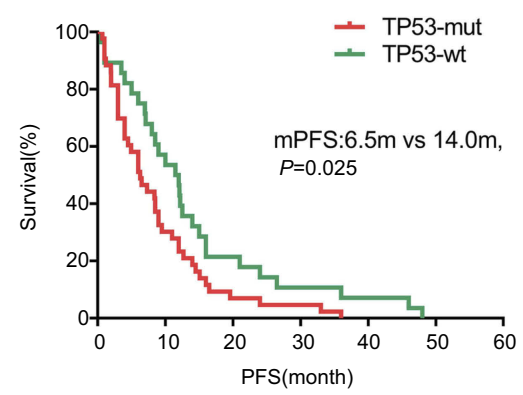

C

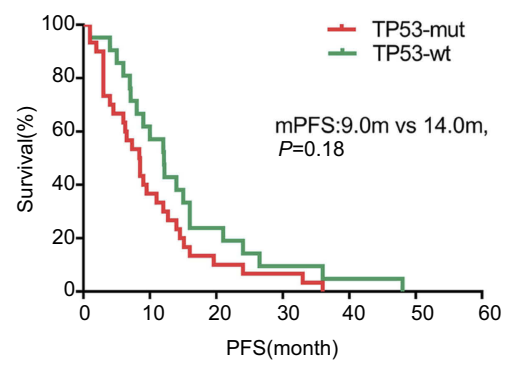

B

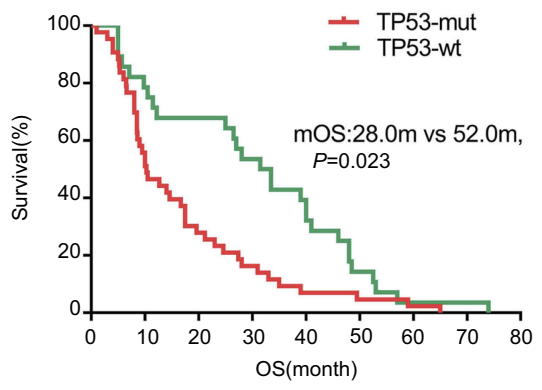

D

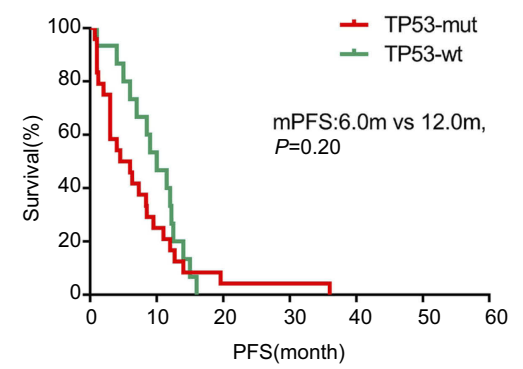

Figure I Kaplan-Meier curves of survival. (A) Comparison of PFS between TP53-mut and TP53-wt patients (N=7I). (B) Comparison of OS between TP53-mut and TP53wt subgroups $(\mathrm{N}=7 \mathrm{I})$. (C) Comparison of PFS between TP53-mut and TP53-wt patients in the subgroup of patients with Dell9/L8585R EGFR mutations ( $\mathrm{N}=5 \mathrm{I}$ ). ( $\mathrm{D}$ ) Comparison of PFS between TP53-mut and TP53-wt patients in the subgroup of patients with first-line TKIs therapy (N=39).

Abbreviations: PFS, progression-free survival; OS, overall survival; NSCLC, non-small cell lung cancer; ECOG, Eastern Cooperative Oncology Group; PS, performance status; mut, mutant; wt, wild type.

in the subgroup of patients with non-first line EGFR-TKI treatment were not acquired.

Given that different types of TP53 genetic mutations tend to have different effects on the functionality of the protein, we classified TP53 mutations into disruptive/nondisruptive, missense/non-missense mutations as well as mutations in different sites based on previous studies (Table 5). ${ }^{5,29-31}$ In contrast to TP53 disruptive mutations, TP53 non-disruptive mutations demonstrate greater potency in shortening $\mathrm{mPFS}$ and OS of the patients (PFS: 6.3 versus 14.0 months, $P=0.028$; HR 2.38, 95\% CI $1.06-5.32, P<0.030$; OS: 35.0 versus 52.5 months, $P=0.008$; HR $5.00,95 \%$ CI $1.38-18.18, P=0.008$ ) when compared with TP53-wt controls (Figure 2A and B, $\underline{\text { Tables S2 }}$ and S3). Similar conclusions could be made on TP53 non-missense mutations: patients with nonmissense mutations showed shorter PFS and OS (mPFS: 6.3 versus 14 months, $P=0.041$; HR $2.01,95 \%$ CI 1.00-4.05, $P=0.046$; mOS: 21.2 versus 52.5 months, $P=0.001$; HR 5.53, 95\% CI 1.79-16.95 $P=0.001)$ than that of TP53-wt cases (Figure 2C and D, Tables S2 and $\underline{\mathrm{S}} 3$ ).

Then, we further investigated the effects of TP53 mutations on different sites on patients' prognosis. Among
TP53 exons 5-8 which encode the DBD region of protein p53, mutation on exon 7 shortens patients' prognosis in the highest degree compared with TP53-wt controls (mPFS: 5.0 versus 14.0 months, $P=0.002$, HR $3.98,95 \%$ CI 1.53-10.31, $P=0.002$; mOS: 14.0 versus 52.5 months, $P=0.008 ; \quad$ HR $5.29, \quad 95 \%$ CI $1.32-20.83, \quad P=0.009$ ) (Figure 2G and $\mathrm{H}$; Tables $\mathrm{S} 4$ and $\underline{\mathrm{S} 5}$ ). In addition, mutations on exon 6 also lead to poor OS and marginally shortened PFS than patients in control group (mPFS: 6.5 versus 14.0 months, $P=0.211$, HR $1.85,95 \%$ CI $0.68-5.03$, $P=0.225$; mOS: 39.0 versus 52.5 months, $P=0.036$; HR $4.03,95 \%$ CI $0.98-16.67, P=0.037$ ) (Figure $2 \mathrm{E}$ and $\mathrm{F}$; Tables S4 and S5). It is worth noting that TP53 mutations on exons other than exons $5-8$, which was in relatively less relation with the principal mechanism of the tumorsuppressing function of protein $\mathrm{p} 53$, also significantly reduced patients' survival by 10 months in mPFS and 37.9 months in mOS than the TP53-wt controls (mPFS: 4.0 versus 14.0 months, $P=0.007$; HR $3.13,95 \%$ CI 1.30-7.52, $P=0.008$; mOS: 14.6 versus 52.5 months, $P=0.002$; HR $8.93,95 \%$ CI $1.70-47.62, \quad P=0.002$ ) (Figure 2I and J; Tables S4 and $\underline{\mathrm{S} 5}$ ).

Among 51 patients with EGFR sensitive mutations receiving EGFR-TKI treatment, 25 had exon 19 deletions and 26 had 
Table 4 Comparison of PFS and OS of patients stratified based on different characteristics

\begin{tabular}{|c|c|c|c|c|c|c|c|}
\hline & \multirow[t]{2}{*}{$\mathbf{N}(\%)$} & \multicolumn{3}{|c|}{ PFS (month) } & \multicolumn{3}{|c|}{ OS (month) } \\
\hline & & Median & $95 \% \mathrm{Cl}$ & $\begin{array}{l}\text { Log- } \\
\text { rank } \\
\text { P-value }\end{array}$ & Median & $95 \% \mathrm{Cl}$ & $\begin{array}{l}\text { Log- } \\
\text { rank } \\
\text { P-value }\end{array}$ \\
\hline \multicolumn{8}{|l|}{ Overall } \\
\hline TP53-mut & $43(60.7)$ & 6.5 & $3.44-9.56$ & $0.025^{*}$ & 28.0 & $8.74-47.26$ & $0.023^{*}$ \\
\hline TP53-wt & $28(39.4)$ & 14.0 & $9.43-18.60$ & & 52.0 & $35.63-69.37$ & \\
\hline \multicolumn{8}{|c|}{ Dell9/L858R or uncommon mutations } \\
\hline Dell9/L858R & $5 I(7 \mid .8)$ & 12.0 & $8.76-15.24$ & 0.067 & 52.5 & $37.12-67.88$ & $0.001 *$ \\
\hline Uncommon mutations & $20(28.2)$ & 5.0 & $2.84-7.06$ & & 14.0 & $0.00-38.31$ & \\
\hline \multicolumn{8}{|l|}{ Treatment line } \\
\hline First line & $39(54.9)$ & 14.5 & $6.95-22.05$ & 0.055 & 40.0 & $26.0-51.95$ & 0.567 \\
\hline Non-first line & $32(45.1)$ & 8.4 & $5.29-11.52$ & & 39.0 & $20.11-59.89$ & \\
\hline \multicolumn{8}{|c|}{ Subgroup of classic Dell9/L858R mutations } \\
\hline TP53-mut & $30(58.8)$ & 9.0 & $4.70-13.30$ & 0.181 & NA & NA & 0.057 \\
\hline TP53-wt & $2 I(4 \mid .2)$ & 14.0 & $10.05-17.95$ & & 39.0 & $|5.29-62.7|$ & \\
\hline \multicolumn{8}{|c|}{ Subgroup of uncommon mutations } \\
\hline TP53-mut & $13(65.0)$ & 4.0 & $0.54-7.46$ & 0.124 & 10.5 & $9.16-11.84$ & 0.137 \\
\hline TP53-wt & $7(35.0)$ & 8.5 & NA & & 40.0 & $1.98-78.02$ & \\
\hline \multicolumn{8}{|c|}{ Subgroup of first line treatment } \\
\hline TP53-mut & $24(6 \mid .5)$ & 6.0 & $2.42-9.58$ & 0.160 & 21.2 & NA & 0.398 \\
\hline TP53-wt & $15(38.5)$ & 12.0 & $6.70-12.30$ & & 40.0 & $0-46.00$ & \\
\hline \multicolumn{8}{|c|}{ Subgroup of non-first line treatment } \\
\hline TP53-mut & $19(59.4)$ & 9.0 & $0-18.66$ & 0.072 & NA & NA & 0.095 \\
\hline TP53-wt & $13(4 \mid .6)$ & 21.0 & $4.60-37.44$ & & 28.0 & $2.02-53.98$ & \\
\hline \multicolumn{8}{|c|}{$\begin{array}{l}\text { Comparison of PFS and OS between patients with } \\
\text { exon } 19 \text { deletion and L858R point mutations }\end{array}$} \\
\hline Dell9 & $25(49.02)$ & 14.0 & $8.44-19.56$ & 0.730 & 59.0 & NA & 0.286 \\
\hline L858R & $26(50.98)$ & 9.5 & $5.77-13.23$ & & 39.0 & II.25-66.75 & \\
\hline \multirow{2}{*}{\multicolumn{8}{|c|}{$\begin{array}{l}\text { Comparison of PFS and OS between patients with } \\
\text { Dell 9/TP53-mut and L858R/TP53-mut genotypes }\end{array}$}} \\
\hline & & & & & & & \\
\hline Dell9/TP53-mut & $12(40.00)$ & 11.0 & $1.54-20.46$ & 0.640 & 59.0 & NA & $0.56 \mathrm{I}$ \\
\hline L858R/TP53-mut & $18(60.00)$ & 9.0 & $6.47-11.53$ & & 39.0 & $12.5-65.50$ & \\
\hline
\end{tabular}

Note: $* P<0.05$.

Abbreviations: PFS, progression-free survival; OS, overall survival

L858R mutations. Compared to patients with EGFR L858R mutations, patients with exon 19 deletions showed longer survival (mPFS: 14.0 versus 9.5 months, $P=0.730$; mOS: 59.0 versus 39.0 months, $P=0.286$ ). On further investigation, we found that 12 patients with Del19/TP53 concurrent mutations $(\mathrm{N}=12)$ also demonstrated longer mPFS (11.0 versus 9.0 months, $P=0.640$ ) and mOS (59.0 versus 39.0 months, $P=0.561)$ compared with that of 18 patients with $\mathrm{L} 858 \mathrm{R} /$ TP53 concurrent mutations $(\mathrm{N}=18)$. However, no significant difference of characteristics (mutation subtype or mutated exons or mutation frequency) of TP53 mutations was identified between these two subgroups of patients (Table S6).

In our study, only $54.9 \%$ (39/71) of all NSCLC patients received first-generation TKIs as first-line therapy. The overall DCR and ORR of the 71 patients were lower than that of the TP53-wt controls (DCR: $76.7 \%$ versus $89.3 \%, P=0.160$; ORR: $25 \%$ versus $28 \%, P=0.374)$. We compared DCR in different TP53 mutational sites with TP53-wt controls in detail (Table S7). However, no difference with statistical significance was observed. 
Table 5 TP53 mutational status based on sites and subtypes

\begin{tabular}{|l|l|l|}
\hline & Exon & Number of patients (N=43) (\%) \\
\hline Mutations on DNA binding domain (N=32, 74.42\%) & Exon 5 & $10(23.25)$ \\
& Exon 6 & $6(13.95)$ \\
& Exon 7 20.93$)$ \\
& Exon 8 & $7(16.28)$ \\
\hline Mutations on other sites (N=II, 25.58\%) & Exon 4 & $6(13.95)$ \\
& Intron 9 & $1(2.33)$ \\
& Exon 10 & $3(6.98)$ \\
\hline Missense/non-missense mutation & Exons I+3 & $\mathrm{I}(2.33)$ \\
\hline Disruptive/non-disruptive & Missense & $27(62.79)$ \\
& Non-missense mutation & $16(37.21)$ \\
\hline
\end{tabular}

\section{Discussion}

In the present study, TP53 mutations were the most common concurrent mutation in Chinese patients with EGFR mutant NSCLC. The frequency of EGFR/TP53 concurrent mutation was $60.6 \%$, which was consistent with previous reports showing percentages of $30-65 \%$ in adenocarcinoma. $^{1,16-18}$ Several reports have suggested that concurrent TP53 alterations are associated with a lower likelihood of response to EGFR-TKIs, and a shorter OS. ${ }^{19-22}$ However, conclusions remain controversial either due to the small size of studies or a lackOS of statistically significant differences in survival between TP53-mutated and TP53-wild type NSCLC patients. In our study, TP53 concurrent mutations were associated with a markedly shorter time to progression and OS on initial EGFR-TKIs, as well as reduced DCR and ORR to TKI therapy. In addition, we found that nondisruptive mutations of the $\mathrm{p} 53$ gene are an independent factor of shorter survival in advanced NSCLC with a possible predictive negative value of response to TKIs; the same conclusions could be seen in two previously published studies. ${ }^{30,31}$ However, the molecular pathogenesis is still unclear. It was hypothesized by one study that the GOF (gain of functionality) activities of p53 protein, which most likely resulted from TP53 non-disruptive mutations, could promote an aggressive behavior of the tumor after progression. ${ }^{27}$ Moreover, we found that TP53 missense mutations were associated with a poorer response to TKI therapy than non-missense mutations. However, a study by Labbé et al showed thatNSCLCpatients with TP53 missense mutations, instead of non-missense mutations, had significantly shorter PFS with first-line EGFR-TKI therapy, ${ }^{21}$ which was contradictive to our finding. In fact, as far as we know, only the study by Labbé et $\mathrm{al}^{21}$ and ours have investigated the potential value of TP53 missense or non-missense mutations on the prognosis of NSCLC patients with EGFR-TKI treatment up to now. However, in Labbé's study, only 17 patients had TP53 missense mutations. ${ }^{21}$ In our study, 27 patients were identified as TP53 missense-mutated. The numbers of patients with a TP53 missense mutation in both studies were relatively small. Therefore, larger cohort analyses are necessary for further investigation on this point.

It has been suggested by several studies that in advanced NSCLC, patients with exon 19 deletions showed better prognosis and longer survival than those with exon 21 L858R point mutations when treated with EGFR-TKIs (gefitinib, erlotinib or afatinib). ${ }^{32-34}$ For example, in a study by Kuiper et al, ${ }^{32}$ mOS of patients with exon 19 deletions with first-line EGFR-TKI therapy was 7.2 months longer than that of patients with exon $21 \mathrm{~L} 858 \mathrm{R}$ point mutations. In our study, longer survival and better responsiveness to EGFR-TKI treatment were observed in both the subgroup of patients with EGFR exon 19 deletions and the subgroup of patients with Del19/TP53 concurrent mutations than those with exon 21 L858R point mutations and L858R/TP53 concurrent mutations, respectively. However, no significant difference of TP53 mutations was identified between these two subgroups of patients, indicating that TP53 concurrent mutations might not be the underlying reason for different responsiveness to EGFR-TKI therapy between patients with EGFR exon 19 deletions and exon 21 L858R point mutations. 
A
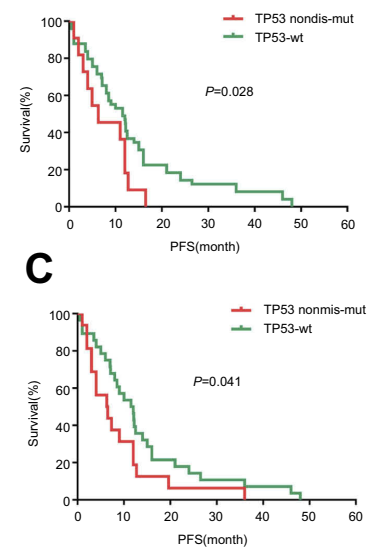

E

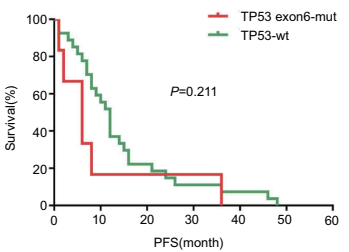

G

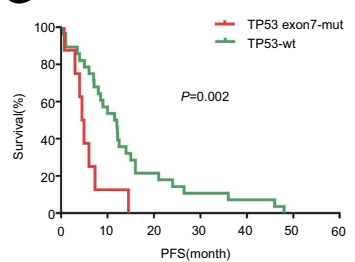

I

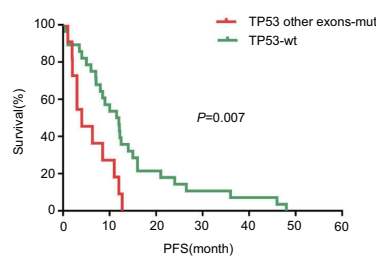

B

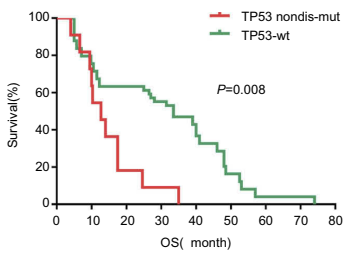

D
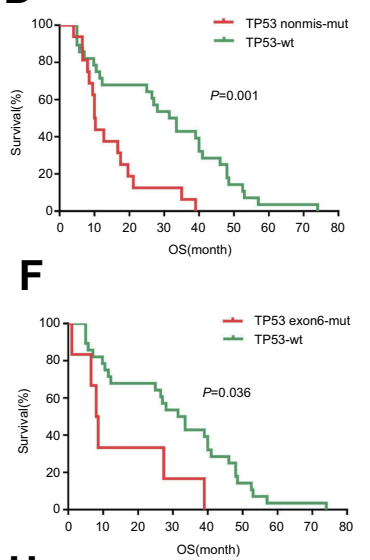

$\mathbf{H}$

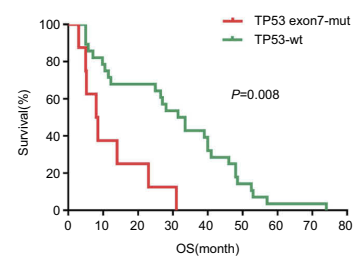

$\mathbf{J}$

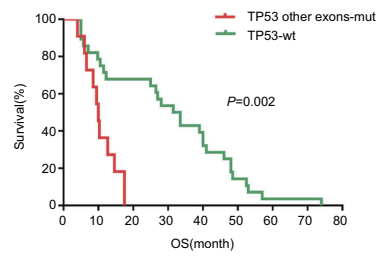

Figure 2 Kaplan-Meier curves of survival. (A and B) Comparison of PFS/OS between patients with TP53 non-disruptive mutations $(\mathrm{N}=\mathrm{II})$ and TP53 wild type cases $(N=28)$. ( $(C$ and $\mathbf{D})$ Comparison of PFS/OS between patients with TP53 nonmissense mutations $(\mathrm{N}=16)$ and TP53 wild type cases $(\mathrm{N}=28)$. ( $E$ and $\mathbf{F}$ ) Comparison of PFS/OS between patients with TP53 mutations in exon $6(\mathrm{~N}=6)$ and TP53 wild type cases $(\mathrm{N}=28)$. ( $(\mathbf{G}$ and $\mathbf{H})$ Comparison of PFS/OS between patients with TP53 mutations in exon $7(\mathrm{~N}=9)$ and TP53 wild type cases $(\mathrm{N}=28)$. (I and J) Comparison of PFS/OS between patients with TP53 mutations in exons other than 5-8 $(\mathrm{N}=\mathrm{II})$ and TP53 wild type cases $(\mathrm{N}=28)$.

Abbreviations: PFS, progression-free survival; OS, overall survival.

In terms of the specific impact of TP53 mutations in various sites on patients' response to TKIs, we found that patients with TP53 mutations in exon 6, exon 7 and exons in the non-DBD region showed obviously poor outcomes with TKI therapy. However, a study by Canale et al suggested that TP53 exon 8 mutations were associated with a significantly lower DCR and shorter survivals, but data were only significant for patients with

EGFR exon 19 deletion. ${ }^{19}$ In our study, $7 / 43$ (16.28\%) patients were TP53 exon 8 mutated, although no difference was observed in PFS between patients with TP53 exon 8 mutations and TP53 wild type cases, whereas a slightly shorter mOS was identified in the former subgroup of patients (mPFS: 52.5 versus 59.0 months, $P=0.908$ ). However, in our study, only 3 patients were EGFR-Del19/TP53-exon 8 mutated and 3 patients were EGFR-L858R/TP53-exon 8 mutated; these numbers were too small to evaluate the effect of TP53 concurrent mutations in relation to different EGFR mutations. In addition, Jiao et al reported that patients with exon 4, exon 6, mutation of an unknown site of TP53 demonstrated worse prognosis than exon 5, exon 7 , exon 8 and exon 9 mutated patients. ${ }^{22}$ However, different from our study, Jiao et al generally investigated the prognostic value of TP53 and its correlation with EGFR mutation in patients with advanced NSCLC, while the specific impact of TP53 concurrent mutation on patients' response to EGFR-TKI treatment was not discussed. Limited by the small number of patients in our study, more dedicated efforts are needed to clarify the biologic effects of mutations in different TP53 exons on disease course and drug responsiveness to TKIs.

In particular, patients with TP53 concurrent mutations showed a much higher proportion of short-term responders (PFS $<3$ months, $P=0.035$; OS $<6$ months, $P=0.26$ ) than the TP53 wild type controls, indicating that TP53 mutations might be involved in primary resistance to TKIs. Several studies also indicated that TP53 mutations were commonly found in non-responders or short-term responders to TKIs. ${ }^{16,24-26}$

However, the underlying mechanism of why EGFR/ TP53 concurrent mutations resulted in reduced sensitivities to TKIs in patients with advanced NSCLC it is still unclear. A preclinical study reported that TP53 gene mutations can reduce gefitinib-induced cell apoptosis and that wild type TP53 could enhance gefitinib-induced growth inhibition and apoptosis by regulation of Fas (factorassociated suicide) signal pathway in NSCLC cell line. ${ }^{35}$ Another study suggested that TP53 and PTEN knockdown may promote epithelial-to-mesenchymal transition by activating pro-inflammatory interleukin-6/STAT3/nuclear factor-kB signaling pathway in highly metastatic cancer stem cells, which indicated a potential relationship between resistance to TKIs and inflammatory activities. ${ }^{36}$ In addition, the histologic transformation of EGFR mutant lung adenocarcinoma into small-cell lung cancer has been 
described as one of the major resistant mechanisms for TKIs. It was reported that TP53 inactivation and RB1 loss might be early events of small cell transformation which would generate resistance to TKIs. ${ }^{37,38}$

To date, there are no approved agents that specifically target TP53 mutations in NSCLC. It is suggested that AZD1775 (a Wee-1 inhibitor) is potential anticancer therapy against the TP53-mut NSCLC. ${ }^{39}$ In recent years, coexisting alterations like amplification of ERBB2 or MET, mutations in TP53, primary EGFR T790M mutations, and BRAF fusions were claimed to portend poorer outcomes in patients with EGFR-mutant NSCLC by an increasing number of studies. Therefore, concurrent mutations should be taken into consideration to guide the selection of combination treatment strategies implemented at treatment onset for targeted therapy of patients.

In summary, our study indicated that TP53 mutations might function as an independent predictor of poor outcome in Chinese patients with advanced EGFR mutationpositive NSCLC and treated with EGFR-TKIs, and might also be involved in the primary resistance to EGFR-TKIs, especially those with non-missense mutations and nondisruptive mutations, and mutations on exon 6 , exon 7 and non-DBD region. To the best of our knowledge, this is the first study to have analyzed the association between TP53 mutations and response to TKIs in advanced EGFRmutant Chinese NSCLC patients with EGFR mutations. These findings have to be confirmed on larger patient populations in the future.

\section{Ethics statement}

This study was approved by the medical ethics committee at the Affiliated Hospital of Qingdao University and was carried out in accordance with the principles of the Declaration of Helsinki. All patients in our study provided written informed consent.

\section{Acknowledgments}

This work was supported by grants from Taishan Scholar Foundation of Shandong Province (No.tshw 201502061 to Xiaochun Zang), Qingdao People's Livelihood Science and Technology Program (16-6-2-3-nsh to Xiaochun Zhang), and Chinese Postdoctoral Science Foundation (2017M622143 to Helei Hou).

\section{Disclosure}

The authors report no conflicts of interest in this work.

\section{References}

1. Forbes SA, Bindal N, Bamford S, et al. COSMIC: mining complete cancer genomes in the Catalogue of Somatic Mutations in Cancer. Nucleic Acids Res. 2011;39:945-950. doi:10.1093/nar/gkq929

2. Hainaut P, Hollstein M. p53 and human cancer: the first ten thousand mutations. Adv Cancer Res. 2000;77:81-137.

3. Cho Y, Gorina S, Jeffrey PD, et al. Crystal structure of a p53 tumor suppressor-DNA complex: understanding tumorigenic mutations. Science. 1994;265:346-355. doi:10.1126/science.8023157

4. Kato S, Han SY, Liu W, et al. Understanding the function-structure and function-mutation relationships of $\mathrm{p} 53$ tumor suppressor protein by high-resolution missense mutation analysis. Proc Natl Acad Sci U S A. 2003;14:8424-8429. doi:10.1073/pnas.1431692100

5. Olivier M, Hainaut P. TP53 mutation patterns in breast cancers: searching for clues of environmental carcinogenesis. Semin Cancer Biol. 2001;11:353-356. doi:10.1006/scbi.2001.0390

6. Sylvanie S, Marie PK, Jean CB, et al. Uncovering the role of $p 53$ splice variants in human malignancy: a clinical perspective. OncoTargets Ther. 2013;7:57-68.

7. Fukuoka $\mathrm{M}, \mathrm{Wu} \mathrm{Y}$, Thongprasert $\mathrm{S}$, et al. Biomarker analyses and final overall survival results from a phase III, randomized, open-label, first-line study of gefitinib versus carboplatin/paclitaxel in clinically selected patients with advanced non-small-cell lung cancer in Asia (IPASS). $J$ Clin Oncol. 2011;29:2866-2874. doi:10.1200/ JCO.2010.33.4235

8. Han J, Park K, Kim S, et al. First-SIGNAL: first-line single-agent iressa versus gemcitabine and cisplatin trial in never smokers with adenocarcinoma of the lung. J Clin Oncol. 2012;30:1122-1128. doi:10.1200/JCO.2011.36.8456

9. Yang JCH, Wu YL, Schuler M, et al. Afatinib versus cisplatin-based chemotherapy for EGFR mutation-positive lung adenocarcinoma (LUX-Lung 3 and LUX-Lung 6): analysis of overall survival data from two randomised, phase 3 trials. Lancet Oncol. 2015;16:141-151. doi:10.1016/S1470-2045(14)71173-8

10. Sequist LV, Yang JC, Yamamoto N, et al. Phase III study of afatinib or cisplatin plus pemetrexed in patients with metastatic lung adenocarcinoma with EGFR mutations. J Clin Oncol. 2013;31:3327-3334. doi:10.1200/JCO.2013.49.0219

11. Awad MM, Shaw AT. ALK inhibitors in non-small cell lung cancer: crizotinib and beyond. Clin Adv Hematol Oncol. 2014;12:429-439.

12. Sequist LV, Waltman BA, Dias-Santagata D, et al. Genotypic and histological evolution of lung cancers acquiring resistance to EGFR inhibitors. Sci Transl Med. 2011;3:75ra26. doi:10.1126/ scitranslmed.3002003

13. Maemondo M, Inoue A, Kobayashi K, et al. Gefitinib or chemotherapy for non-small-cell lung cancer with mutated EGFR. $N$ Engl $J$ Med. 2010;362:2380-2388. doi:10.1056/NEJMoa0909530

14. Spaans JN, Goss GD. Drug resistance to molecular targeted therapy and its consequences for treatment decisions in non-small-cell lung cancer. Front Oncol. 2014;4:190. doi:10.3389/fonc.2014.00190

15. Eng J, Woo KM, Sima CS, et al. Impact of concurrent PIK3CA mutations on response to EGFR tyrosine kinase inhibition in EGFR-mutant lung cancers and on prognosis in oncogene-driven lung adenocarcinomas. $J$ Thorac Oncol. 2015;10:1713-1719. doi:10.1097/JTO.0000000000000671

16. Yu H, Suzawa K, Jordan EJ, et al. Concurrent alterations in EGFR-mutant lung cancers associated with resistance to EGFR kinase inhibitors and characterization of MTOR as a mediator of resistance. Clin Cancer Res. 2018;24:3108-3118. doi:10.1158/10780432.CCR-17-2961

17. Scoccianti C, Vesin A, Martel G, et al. Prognostic value of TP53, KRAS and EGFR mutations in non-small cell lung cancer: the EUELC cohort. Eur Respir J. 2012;40:177-184. doi:10.1183/ 09031936.00097311 
18. Halvorsen AR, Silwal-Pandit L, Meza-Zepeda LA, et al TP53Mutation spectrum in smokers and never smoking lung cancer patients. Front Genet. 2016;7:85. doi:10.3389/fgene.2016.00085

19. Canale M, Petracci E, Delmonte A, et al. Impact of TP53 mutations on outcome in EGFR-mutated patients treated with first-line tyrosine kinase inhibitors. Clin Cancer Res. 2017;23:2195-2202. doi:10.1158/ 1078-0432.CCR-16-0966

20. Vanderlaan PA, Rangachari D, Mockus SM, et al. Mutations in TP53, PIK3CA, PTEN and other genes in EGFR mutated lung cancers: correlation with clinical outcomes. Lung Cancer. 2017;106:17-21. doi:10.1016/j.lungcan.2017.01.011

21. Labbé C, Cabanero M, Korpanty GJ, et al. Prognostic and predictive effects of TP53 co-mutation in patients with EGFR-mutated non-small cell lung cancer (NSCLC). Lung Cancer. 2017;111:23-29. doi:10.1016/j.lungcan.2017.06.014

22. Jiao X, Qin B, You P, et al. The prognostic value of TP53 and its correlation with EGFR mutation in advanced non-small cell lung cancer, an analysis based on cBioPortal data base. Lung Cancer. 2018;123:70-75. doi:10.1016/j.lungcan.2018.07.003

23. Lim SM, Kim HR, Cho EK, et al. Targeted sequencing identifies genetic alterations that confer primary resistance to EGFR tyrosine kinase inhibitor (Korean Lung Cancer Consortium). Oncotarget. 2016;7:36311-36320. doi:10.18632/oncotarget.8904

24. Bria E, Pilotto S, Amato E, et al. Molecular heterogeneity assessment by next-generation sequencing and response to gefitinib of EGFR mutant advanced lung adenocarcinoma. Oncotarget. 2015;6:12783-12795. doi:10.18632/oncotarget.3727

25. Xu Y, Tong X, Yan J, et al. Short-term responders of non-small cell lung cancer patients to EGFR tyrosine kinase inhibitors display high prevalence of TP53 mutations and primary resistance mechanisms. Transl Oncol. 2018;11:1364-1369. doi:10.1016/j.tranon.2018.08.010

26. Ulivi P, Delmonte A, Chiadini E, et al. Gene mutation analysis in EGFR wild type NSCLC responsive to erlotinib: are there features to guide patient selection? Int J Mol Sci. 2015;16:747-757. doi:10.3390/ ijms 16010747

27. Hou H, Dong L, Zhang C, et al. Targeted next generation sequencing in Chinese colorectal cancer patients guided anti-EGFR treatment and facilitated precision cancer medicine. Oncotarget. 2017;8:105072-105080. doi:10.18632/oncotarget.21349

28. Therasse P, Arbuck SG, Eisenhauer EA, et al. New guidelines to evaluate the response to treatment in solid tumors. European organization for research and treatment of cancer, national cancer institute of the united states, national cancer institute of Canada. J Natl Cancer Inst. 2000;92:205-216. doi:10.1093/jnci/92.3.205
29. Poeta ML, Manola J, Goldwasser MA, et al. TP53 mutations and survival in squamous-cell carcinoma of the head and neck. $N$ Engl J Med. 2007;357:2552-2561. doi:10.1056/NEJMoa073770

30. Molina-Vila MA, Bertran-Alamillo J, Gascó A, et al. Nondisruptive p53 mutations are associated with shorter survival in patients with advanced non-small cell lung cancer. Clin Cancer Res. 2014;20:4647-4659. doi:10.1158/1078-0432.CCR-13-2391

31. Griesinger F, Netchaeva M, Lüers A, et al. P53 non-disruptive mutation is a negative predictive factor in EGFR M+ NSCLC treated with TKI. Ann Oncol. 2016;27:416-454. doi:10.1093/ annonc/mdw383.32

32. Kuiper JL, Hashemi SMS, Thunnissen E, et al. Non-classic EGFR mutations in a cohort of Dutch EGFR-mutated NSCLC patients and outcomes following EGFR-TKI treatment. Brit J Cancer. 2016;115 (12):1504-1512. doi:10.1038/bjc.2016.372

33. Rosell R, Carcereny E, Gervais R, et al. Erlotinib versus standard chemotherapy as first-line treatment for European patients with advanced EGFR mutation-positive non-small-cell lung cancer (EURTAC): a multicentre, open-label, randomised phase 3 trial Lancet Oncol. 2012;13(3):239. doi:10.1016/S1470-2045(12)70140-7

34. Jackman DM, Yeap BY, Sequist LV, et al. Exon 19 deletion mutations of epidermal growth factor receptor are associated with prolonged survival in non-small cell lung cancer patients treated with gefitinib or erlotinib. Clin Cancer Res. 2006;12(13):3908-3914. doi:10.1158/ 1078-0432.CCR-06-0462

35. Jin KR, Yun JC, Ryoo BY, et al. p53 enhances Gefitinib-induced growth inhibition and apoptosis by regulation of Fas in non-small cell lung cancer. Cancer Res. 2007;67:1163-1169. doi:10.1158/00085472.CAN-06-2037

36. Kim G, Ouzounova M, Quraishi AA, et al. SOCS3-mediated regulation of inflammatory cytokines in PTEN and p53 inactivated triple negative breast cancer model. Oncogene. 2015;34:671-680. doi: $10.1038 /$ onc. 2014.4

37. Lee JK, Lee J, Kim S, et al. Clonal history and genetic predictors of transformation into small-cell carcinomas from lung adenocarcinomas. J Clin Oncol. 2017;35:3065-3074. doi:10.1200/ JCO.2016.71.9096

38. Peifer M, Lynnette Fernández C, Sos ML, et al. Integrative genome analyses identify key somatic driver mutations of small-cell lung cancer. Nat Genet. 2012;44:1104-1110. doi:10.1038/ng.2396

39. Ku BM, Bae YH, Koh J, et al. Mutational status of TP53 defines the efficacy of Wee1 inhibitor AZD1775 in KRAS-mutant non-small cell lung cancer. Oncotarget. 2017;8:67526-67537. doi:10.18632/ oncotarget. 18728
Cancer Management and Research

\section{Publish your work in this journal}

Cancer Management and Research is an international, peer-reviewed open access journal focusing on cancer research and the optimal use of preventative and integrated treatment interventions to achieve improved outcomes, enhanced survival and quality of life for the cancer patient.
The manuscript management system is completely online and includes a very quick and fair peer-review system, which is all easy to use. Visit http://www.dovepress.com/testimonials.php to read real quotes from published authors. 